\title{
Odorant responses of Xenopus laevis tadpole olfactory neurons: a comparison between preparations
}

\author{
Ivan Manzini, Florian Peters, Detlev Schild* \\ Physiologisches Institut, Universität Göttingen, Humboldtallee 23, 37073 Göttingen, Germany
}

Received 20 May 2002; received in revised form 22 July 2002; accepted 14 August 2002

\begin{abstract}
We used a slice preparation of the olfactory epithelium of Xenopus laevis tadpoles to record odorant responses of olfactory receptor neurons (ORNs) and compared these to odorant responses recorded in isolated ORNs. The maximum recording time in the slice was considerably longer than in isolated ORNs, which is essential when many odorants are to be tested. No odorant-induced responses could be obtained from isolated ORNs recorded in the on-cell mode, while recordings in the slice (on-cell and whole-cell) as well as previously reported perforated-patch recordings in isolated ORNs of the same species (Vogler and Schild, 1999) were successful, though qualitatively different. In the slice preparation, amino acids as well as an extract from Spirulina algae always induced excitatory responses, while, in a previous study on isolated ORNs, responses were either excitatory or inhibitory. The results of this study show that ORNs obtained using different preparation techniques can give markedly different responses upon the application of odorants. Our experiments indicate that the slice preparation combined with the on-cell configuration of the patchclamp technique is the method of choice for testing many odorants on individual ORNs.
\end{abstract}

(C) 2002 Elsevier Science B.V. All rights reserved.

Keywords: Xenopus laevis; Olfactory receptor neurons; Olfactory mucosa slice

\section{Introduction}

The classical studies on the response behavior of olfactory receptor neurons (ORNs) were performed using extracellular and intracellular recordings (Getchell, 1986). Since then ORNs have primarily been studied using the patch-clamp technique. Odorant responses have been recorded in isolated ORNs (for a review see Schild and Restrepo, 1998), in the epithelium (Frings and Lindemann, 1991) or in slice preparations of the olfactory mucosa (Taniguchi et al., 1996). While slices are less adequate for simultaneous electrophysiological and optical measurements, they have the advantage of retaining both their epithelial polarization as well as the overlying mucous layer. Isolated ORNs usually are obtained by enzymatic dissociation, and this affects not only the integrity of their tight junctions, but also

\footnotetext{
* Corresponding author. Tel.: +49-551-395915; fax: +49-551395923

E-mail address: dschild@gwdg.de (D. Schild).
}

physiological properties such as the spiking properties (Imanaka and Takeuchi, 2001).

Responses to odorants also appear to differ in the different types of preparations. Approximately equal excitatory and inhibitory odorant responses occurred in isolated ORNs of Xenopus laevis tadpoles recorded in the perforated-patch configuration (Vogler and Schild, 1999), while odorant responses recorded in tissue slices of the lateral olfactory cavity of adult Xenopus laevis were exclusively excitatory (Iida and Kashiwayanagi, 1999).

These diverging results could depend either on the developmental stages studied, or alternatively, isolated ORNs could show a response behavior that differs from that of ORNs in the tissue slice. Obviously, the mucus on the mucosal surface and the tight junction barrier between the outside mucous layer and the extracellular compartment of the mucosa are two major differences between the slice preparation and isolated ORNs. Whether or not these differences affect the response behavior of ORNs is important for future studies on 
odorant transduction as well as for the interpretation of past studies on odorant responses of ORNs.

Responses to odorants of isolated ORNs of different species, including Xenopus laevis, are reported to show a marked run-down of their odorant sensitivity (Lucero et al., 1992; Dionne, 1992; Lischka and Schild, 1993), when using the whole-cell configuration of the patch-clamp technique. Testing the responsiveness of ORNs to many odorants is therefore not feasible. The on-cell configuration is less invasive and overcomes this problem, but the spontaneous activity measured in isolated ORNs in this configuration is typically very low or absent (Dionne, 1992; Vogler and Schild, 1999). This in turn hampers the detection of inhibitory responses. Whether or not these limitations exist also in the slice preparation of the olfactory epithelium is unclear.

In the present study we set out to record odorant responses from ORNs in slices of the olfactory epithelium of Xenopus laevis tadpoles and to compare these with recordings from isolated ORNs.

\section{Methods}

\subsection{Tissue dissociation and preparation of isolated ORNs}

ORNs were prepared from the olfactory mucosa of tadpoles (stages 48-54, Nieuwkoop and Faber, 1956) of Xenopus laevis as described previously (Vogler and Schild, 1999). Briefly, after anaesthesia in a mixture of water and ice and subsequent decapitation, the tissue above the mucosa was removed and the mucosa was extirpated and placed into the cell dissociation solution consisting of $109 \mathrm{mM} \mathrm{NaCl}, 2 \mathrm{mM} \mathrm{KCl}, 10 \mathrm{mM}$ glucose and $10 \mathrm{mM}$ HEPES (pH 7.8, $230 \mathrm{mOsmol}$ ) which contained papain (Sigma, Deisenhofen, Germany; $12-$ 15 units per $\mathrm{ml}, 27{ }^{\circ} \mathrm{C}$ ) and $2 \mathrm{mM}$ EDTA. After $3 \mathrm{~min}$ in this solution, the mucosa was transferred into approximately $600 \mu \mathrm{l}$ of cell dissociation solution without papain and EDTA and was mechanically macerated using two pairs of fine forceps. The resulting pieces of mucosa were then triturated with a plastic pipette (2-mm inner diameter), and $100 \mu \mathrm{l}$ of bath solution (see below) was added. Samples of $100-150 \mu 1$ of the cell suspension were stored at $5{ }^{\circ} \mathrm{C}$ on glass coverslips coated with Concanavalin A $(1 \mathrm{mg} / \mathrm{ml})$. The cells were used within 6 $\mathrm{h}$ after preparation. Fig. 1A shows a typical dissociated olfactory neuron of a Xenopus laevis tadpole viewed through Nomarski optics (Axioskop 2, Zeiss, Göttingen, Germany).

\subsection{Slice preparation}

Tadpoles (stages 49-54) were anaesthetized and sacrificed as described above. A block of tissue containing the olfactory mucosa and the olfactory nerves was cut out and kept in the bath solution (see below). The tissue was glued onto the stage of a vibroslicer (VT 1000S, Leica, Bensheim, Germany) and cut horizontally into $120-125 \mu \mathrm{m}$ thick slices. Only one slice per animal was taken for recording. Fig. 1B shows a slice of the olfactory epithelium ('mucosa slice') stained with biocytin/avidin (Nezlin and Schild, 2000) by backfilling the receptor axons from the glomerular layer of the olfactory bulb. The slice was counterstained with propidium iodide (see next section for staining procedures). Fig. 1C and D show the typical shape of ORNs and cilia issuing from dendritic knobs.

Prior to recording, the fresh slices were placed under a grid in a recording chamber (Edwards et al., 1989) and viewed using Nomarski optics (Axioskop 2, Zeiss).

\subsection{Staining of the olfactory mucosa}

Tadpoles were anaesthetized and sacrificed as described above. A block of tissue containing the olfactory mucosa, the olfactory nerves and anterior part of the brain, including the olfactory bulb, was cut out and kept in bath solution (see below). Blocks of tissue were pinned to the bottom of a silicone-covered Petri dish and the tissue above the olfactory bulb was removed. Then $5 \mu \mathrm{l}$ of DMSO was dropped onto the olfactory bulb and crystals of biocytin (Molecular Probes, Eugene, OR) were pinched into the glomerular layer using a fine needle. After $40 \mathrm{~min}$ of nerve backfilling, the blocks were placed into bath solution for $1 \mathrm{~h}$. The tissue blocks were fixed overnight in $4 \%$ formaldehyde in phosphate buffer solution (PBS, pH 7.4), washed in PBS, embedded in 5\% low melting point agarose (Sigma), and sectioned at $70 \mu \mathrm{m}$ on a vibroslicer. Sections were washed in PBS containing $0.2 \%$ Triton $\mathrm{X}-100$ (PBST). The tissue was incubated for $2 \mathrm{~h}$ at room temperature in avidin, ALEXA 488 conjugated (Molecular Probes, 1:200 in PBS with 0.2\% Triton X-100). For staining cell nuclei, the avidin in PBS was washed off the tissue and the sections were incubated for $15 \mathrm{~min}$ in 25 $\mu \mathrm{g} / \mathrm{ml}$ propidium iodide (Molecular Probes) in PBS. Sections were washed in at least 5 changes of PBS and transferred into $60 \%$ glycerol/PBS for at least $1 \mathrm{~h}$, and mounted on slides in $80 \%$ glycerol/PBS. Preparations were viewed and imaged using a laser-scanning confocal microscope (Zeiss LSM 510/Axiovert 100, Jena, Germany).

\subsection{Recording}

Patch electrodes with a tip diameter of 1-2 $\mu \mathrm{m}$ and approximately 7-10 $\mathrm{M} \Omega$ resistance were fabricated from borosilicate glass (1.8-mm outer diameter; Hilgenberg, Malsfeld, Germany) using a two-stage electrode puller (Narishige, Tokyo) from borosilicate glass and firepolished. Pulse protocols, data acquisition and evalua- 
A

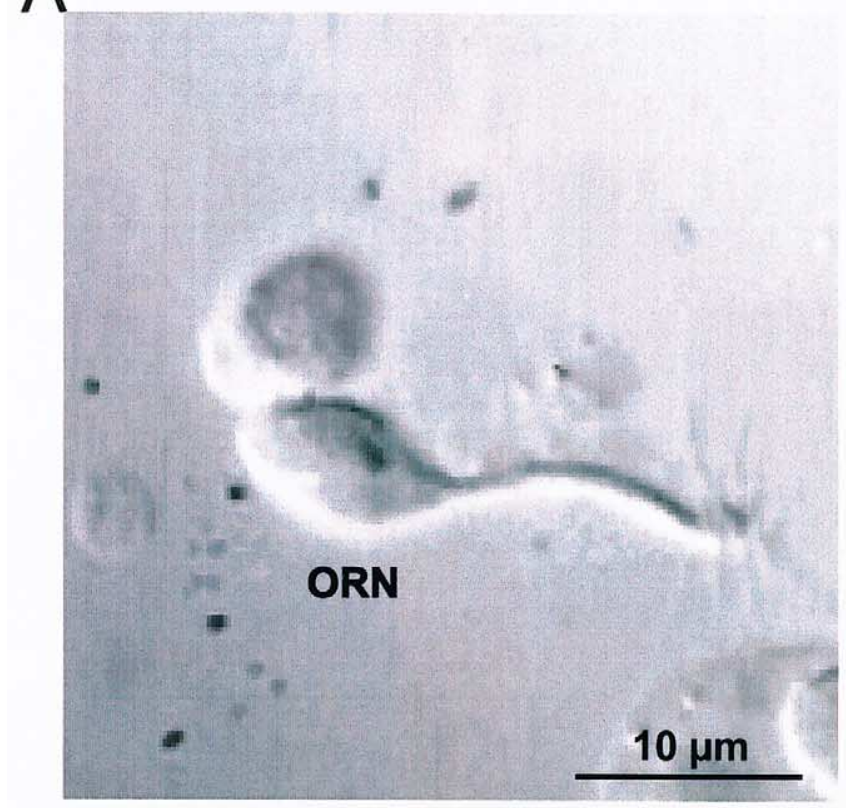

C

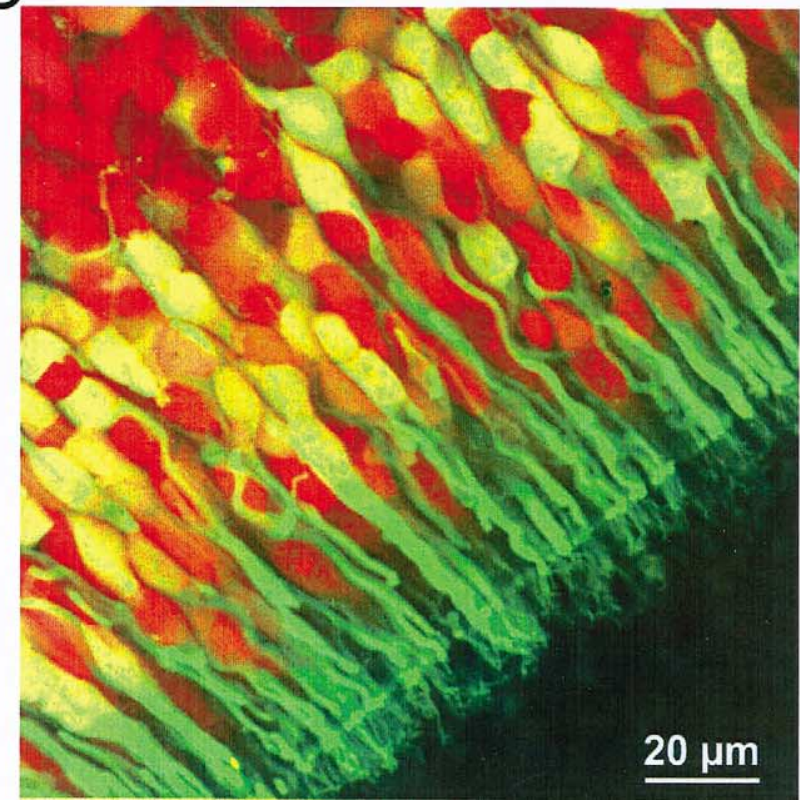

B

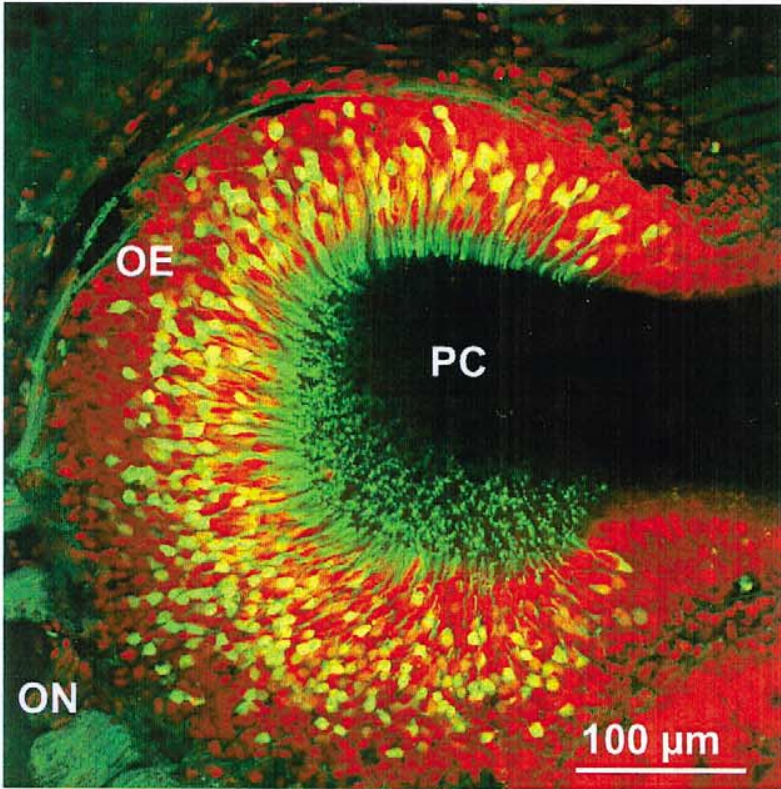

$\mathrm{D}$

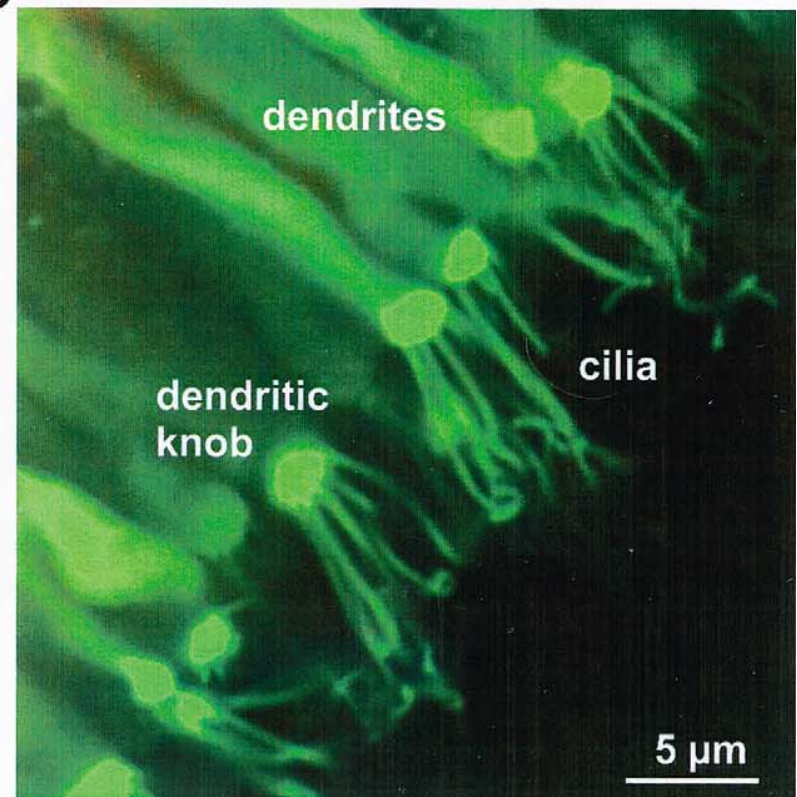

Fig. 1. Isolated receptor neuron (stage 49) and receptor neurons in a slice of the olfactory epithelium (stage 54) of Xenopus laevis tadpoles. (A) Typical ORN with soma, dendrite, dendritic knob and cilia viewed through Nomarski optics. (B) A horizontal overview over the olfactory epithelium (PC, principal cavity; OE, olfactory epithelium and $\mathrm{ON}$, olfactory nerve). The neurons were backfilled through the nerve using biocytin/avidin staining (green fluorescence), and then the slice was counterstained with propidium iodide (red fluorescence). (C) Typical shape of olfactory receptor neurons in a slice. (D) Higher magnification of the apical part of ORNs showing dendrites, dendritic knobs and cilia.

tion programs were written in 'C'. Pulses were delivered from a microcontroller (Schild et al., 1996) to a D/A converter and to the patch-clamp amplifier (EPC7; List, Darmstadt, Germany). Currents and voltages were recorded on video tape using a PCM unit (Instrutech, Elmont, NY). The data were digitized off-line using an 8-pole Bessel filter, an A/D converter and a PC. Further data analysis was performed on a PC under LINUX.

\subsection{Solutions and stimulus application}

The composition of the bath solution was (in $\mathrm{mM}$ ): 98 $\mathrm{NaCl}, 2 \mathrm{KCl}, 1 \mathrm{CaCl}_{2}, 2 \mathrm{MgCl}_{2}, 5$ glucose, $5 \mathrm{Na}-$ pyruvate, 10 HEPES. The pipette solution used for whole-cell and on-cell recordings contained (in $\mathrm{mM}$ ): 2 $\mathrm{NaCl}, 47 \mathrm{KCl}, 2 \mathrm{MgCl}_{2}$, $43 \mathrm{~K}$-gluconate, 10 HEPES, 0.2 EGTA, $2 \mathrm{Na}_{2}$-ATP, $0.1 \mathrm{Na}_{2}$-GTP. All solutions were 
Table 1

Mixtures of volatile stimuli used

\begin{tabular}{ll}
\hline Mixture & Compounds \\
\hline $\mathrm{V}_{1}$ & Citralva, eugenol, l-carvone, geraniol, citronellal \\
$\mathrm{V}_{2}$ & Lilial, lyral, ethylvanillin \\
\hline
\end{tabular}

Mixtures $\mathrm{V}_{1}$ and $\mathrm{V}_{2}$ of volatile odorants (Sklar et al., 1986). $\mathrm{V}_{1}$ and $\mathrm{V}_{2}$ contain odorants believed to stimulate the production of the second messengers cAMP and $\mathrm{IP}_{3}$, respectively. Compounds were applied at $100 \mu \mathrm{M}$ each, except ethylvanillin which was applied at $50 \mu \mathrm{M}$.

adjusted to $\mathrm{pH}$ 7.8. Osmolarities of the bath and pipette solutions were 230 and $190 \mathrm{mOsmol}$, respectively. For odorants, we used two mixtures of volatile stimuli dissolved in bath solution (Table 1), 19 amino acids (AA) (Table 2), and an extract of amphibia food based on Spirulina algae (SP, Mikrozell, Dohse Aquaristik Bonn, Germany).

The algae extract was made by dissolving $0.5 \mathrm{~g}$ SP powder in $100 \mathrm{ml}$ bath solution, centrifuging it at $1000 \times g$ for $5 \mathrm{~min}$ and filtering it through a single use filter $(0.5 \mu \mathrm{m}$ pore size, Minisart, Sartorius AG, Göttingen, Germany).

Citralva, lilial and lyral were purchased from International Flavor \& Fragrances (Hilversum, The Netherlands). The other odorants were from Sigma. The AA were applied either as a mixture of 19 AA, each at a concentration of $200 \mu \mathrm{M}$, or as submixtures (LCN, SCN, BAS, ACID or AROM; Table 2), or as single AA, each at $200 \mu \mathrm{M}$ dissolved in bath solution. The bath solution was applied by gravity feed from a storage syringe through a funnel drug applicator (Schild, 1985) to the recording chamber. The flow from the funnel into the mucosa was $250 \mu \mathrm{l} / \mathrm{min}$. Odorants were pipetted directly into the funnel without stopping the flow. We determined the dilution of the stimulus in the mucosa by putting a confocal volume $(\approx 1 \mathrm{fl})$ of a laser-scanning confocal microscope (Zeiss LSM 510/Axiovert 100), first, in front of the funnel outlet and, second, in front of the epithelial surface and measured the respective fluorescences. For this control measurement we used the fluorescent probe tetramethylrhodamine (Sigma; 500

Table 2

Water-soluble mixtures of $\mathrm{L}$-amino acids

\begin{tabular}{ll}
\hline Mixture & Amino acids \\
\hline LCN & $\begin{array}{l}\text { Proline, valine, leucine, isoleucine, methionine } \\
\text { SCN }\end{array}$ \\
& $\begin{array}{l}\text { Glycine, alanine, serine, threonine, cysteine, asparagine, } \\
\text { glutamine }\end{array}$ \\
BAS & Arginine, lysine, histidine \\
ACID & Glutamate, aspartate \\
AROM & Tryptophane, phenylalanine \\
AA & LCN, SCN, BAS, ACID and AROM \\
\hline
\end{tabular}

Mixtures of L-amino acids following Caprio and Byrd (1984). LCN, long chain neutral AA. SCN, short chain neutral AA. BAS, basic AA. ACID, acidic AA. AROM, aromatic AA.
$\mathrm{nM}$ ) as a 'model of the stimulus'. The dilution factor was $0.91 \pm 0.02$ (mean \pm S.D., $n=7$ ). The minimum interstimulus interval was $1 \mathrm{~min}$.

\section{Results}

We made patch-clamp recordings upon the application of odorants in 102 isolated ORNs and in 298 ORNs in slice preparations. The isolated ORNs and the ORNs in the slice were obtained from 37 and 117 animals, respectively. The measurements in the mucosa slice differed from those in isolated ORNs in recording duration and type of odorant response.

\subsection{Recording duration}

The average recording time of 102 isolated ORNs recorded in the on-cell mode was $6.4 \mathrm{~min}$ and rarely longer than $15 \mathrm{~min}$ (Fig. 2A). All of the isolated ORNs were lost due to seal break, which always happened before the end of the stimulus protocol. Recordings in the slice were categorized in three classes: Some ORNs (106) were lost (seal break) before the end of the experimental protocol (Fig. 2B). Some of the ORNs in this group responded to odorants, but as none of them survived the stimulus protocol it was not possible to determine the complete odorant response profile of these ORNs.

Others (165) did not respond to any of the stimuli tested, and we terminated the recording prior to seal break (Fig. 2C). The recording times of successful experiments, which were stopped after the end of the experimental protocol (27 ORNs), are shown in Fig. 2D. The minimum recording time of successful slice experiments was equal to the maximum recording time of isolated ORNs. The recording time of on-cell recordings in the slice preparation averaged over successful and unsuccessful recordings (Fig. 2B and D) was $16.4 \mathrm{~min}$. The average recording time of the successful recordings (Fig. 2D) was $44.5 \mathrm{~min}$.

\subsection{On-cell mode odorant responses of isolated ORNs}

In the on-cell mode of the patch-clamp technique (Hamill et al., 1981), individual action potentials are reflected by a biphasic, mixed capacitative/ohmic current associated with the time course of the membrane potential during an action potential (Lynch and Barry, 1989). In these experiments we clamped the pipette voltage to $0 \mathrm{mV}$. One hundred and two isolated ORNs (stages 48-54) were tested for odorant responses in the on-cell mode using (i) a mixture of AA (36 cells), (ii) single AA (82 cells), or (iii) the mixtures of volatile odorants $V_{1}$ and $V_{2}(21$ cells). Only ORNs that satisfied a number of morphological criteria were selected for 

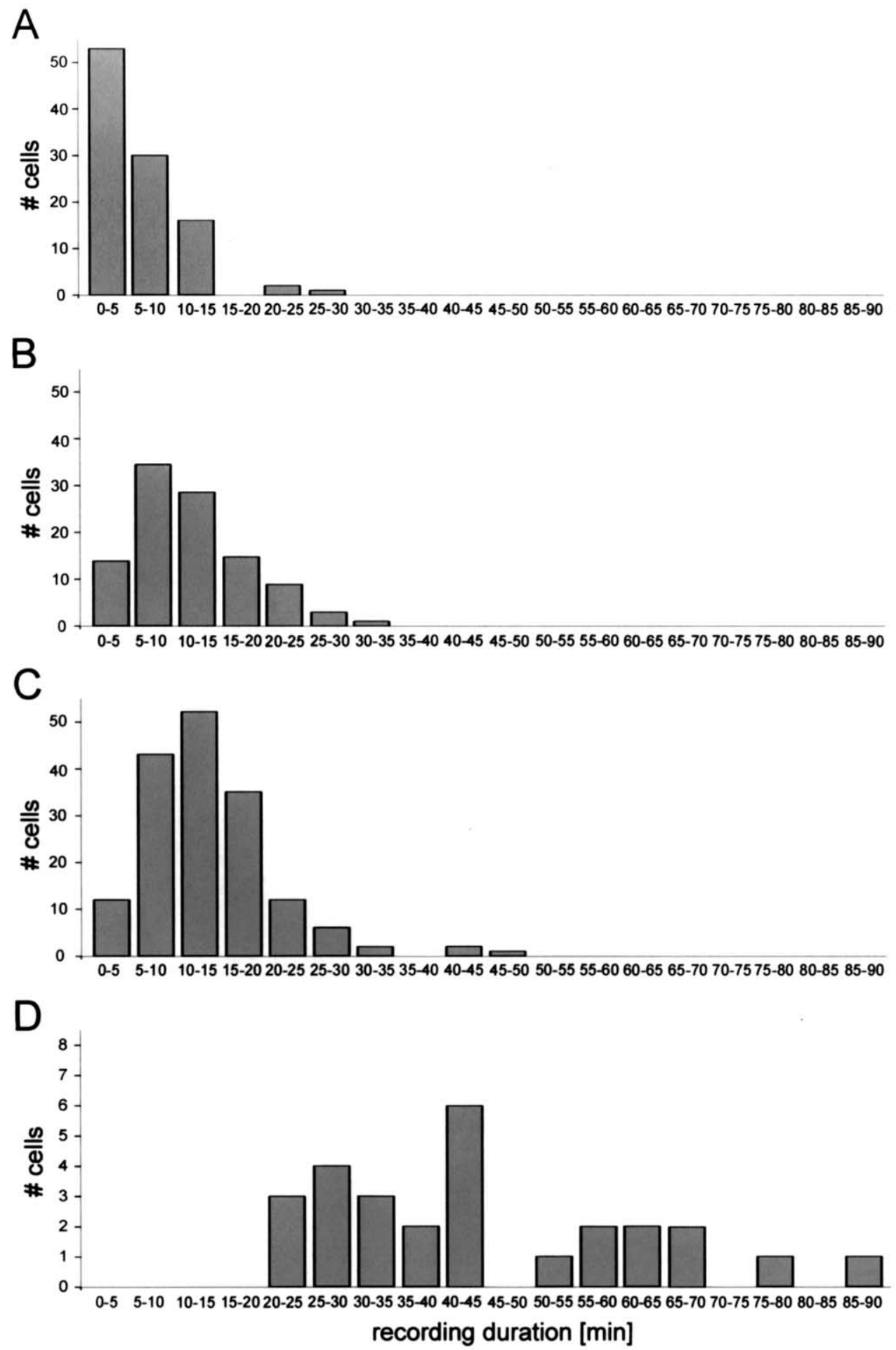

Fig. 2. Histograms of the recording times of isolated ORNs and ORNs in the mucosa slice. (A) Recording times of 102 isolated ORNs. The recording times of ORNs in the slice are plotted in B, C and D. (B) 106 ORNs lost (seal break) before the end of the experimental protocol, (C) 165 ORNs did not respond to any of the stimuli applied, and therefore the recording was terminated, (D) Recording times of the successful experiments stopped at the end of the experimental protocol (27 cells). Recording time was measured from seal formation to the end of the recording.

recording. They had to have an intact soma and dendrite as well as a dendritic knob and at least 4 visible cilia. $36 \%$ of these ORNs showed a spontaneous spiking rate of 3 spikes per second on the average. The remaining ORNs had no spontaneous spiking rate. None of these cells responded to any of the stimuli. 


\subsection{On-cell mode odorant responses of ORNs in the slice}

We made recordings from 111 ORNs (stages 49-54) in the mucosa slice using the on-cell mode. All of these cells were tested for responsiveness to AA. Seventeen ORNs $(15.3 \%)$ responded to AA. Fig. 3 shows an example of a cell which responded to the AA mixture, to a mixture of basic amino acids (BAS), to L-arginine and to L-lysine. The cell did not respond to the third BAS, L-histidine, nor to the mixtures of acidic (ACID) or aromatic (AROM) AA, nor to the short chain neutral (SCN) or long chain neutral (LCN) AA.

The overall effect of all responses on the spiking rate was excitatory though the spontaneous spiking activity was always sufficiently high (Fig. 4) to observe a decrease if it should have occurred. The amino acidsensitive ORNs had spontaneous spiking rates from 2 to 13 spikes/s. No correlation between odorant-sensitivity and spontaneous activity could be observed.

None of the cells tested for volatile odorants responded to the mixtures $\mathrm{V}_{1}$ or $\mathrm{V}_{2}$.

In most experiments, at least some stimuli were applied repeatedly to assess the reproducibility of the recording. The responses to L-arginine shown in Fig. 5 are representative for all odorant-sensitive ORNs. This and other experiments indicate that the responses to AA remain stable over a long period of time.

\subsection{Whole-cell mode odorant responses of ORNs in the slice}

As odorant responses of isolated ORNs when recorded in the whole-cell mode show a fast run-down
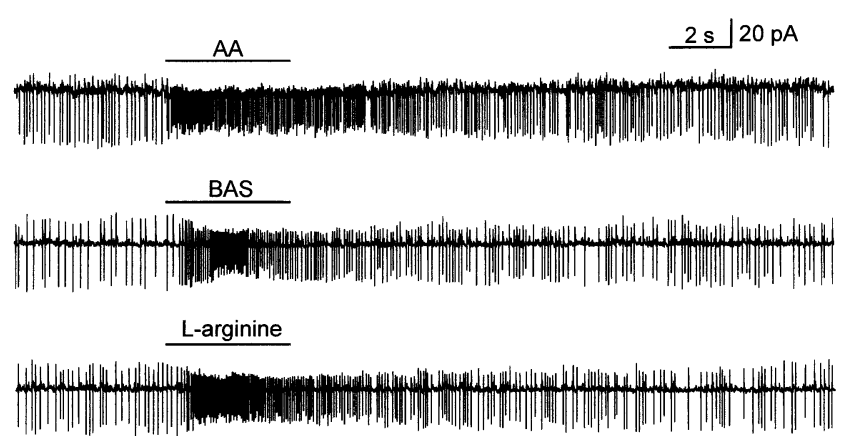

L-lysine

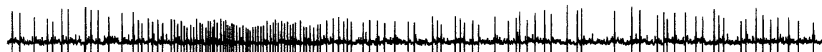
21

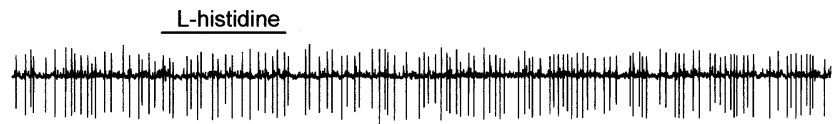

Fig. 3. Odorant responses of an ORN in the mucosa slice recorded from a tadpole in the on-cell mode (stage 53). Spontaneous activity and stimulus-dependent changes are representative for all recorded ORNs.

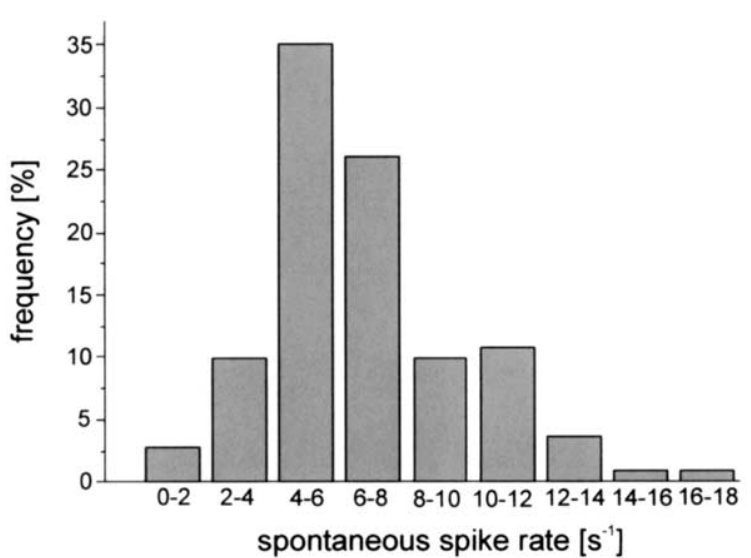

Fig. 4. Frequency histogram of spontaneous spiking activities of ORNs in the mucosa slice. 111 ORNs (stages 49-54) recorded in the on-cell mode were evaluated for this histogram.
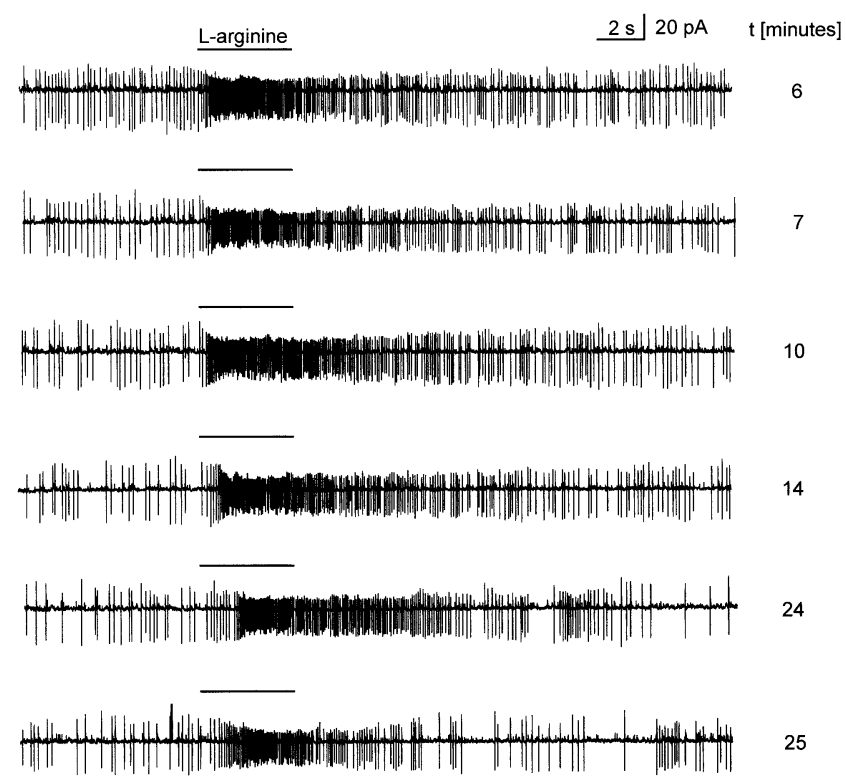

Fig. 5. Reproducibility of odorant responses over the time of an experiment. The response to L-arginine of the ORN shown in Fig. 3 is given here. Responses were taken at the times indicated at the right of the respective trace.

(Lucero et al., 1992; Dionne, 1992; Lischka and Schild, 1993), we did not use this configuration with isolated ORNs. Instead we made whole-cell recordings in the mucosa slice from 187 ORNs (stages 49-54). AA, algae extract and volatile odorants were applied as stimuli. Ten of 116 ORNs $(8.6 \%)$ that were tested for responsiveness to AA showed a response. Eighty-six ORNs were tested for responsiveness to both AA and algae extract (SP). Five ORNs responded to both AA and SP, while 4 responded to AA but not to SP, and 26 responded to SP but not to AA. Fifty-one of the ORNs did not respond to either stimulus.

Odorant application induced receptor potentials with superimposed action potentials (Fig. 6A and B). Thresh- 
A

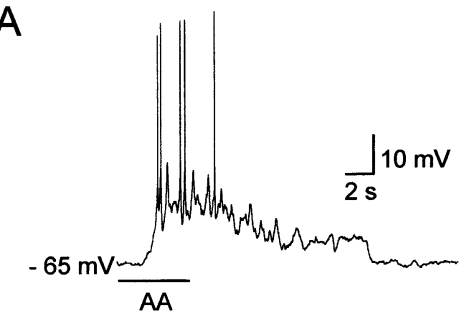

B

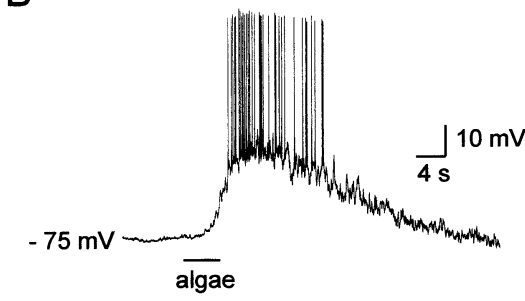

C

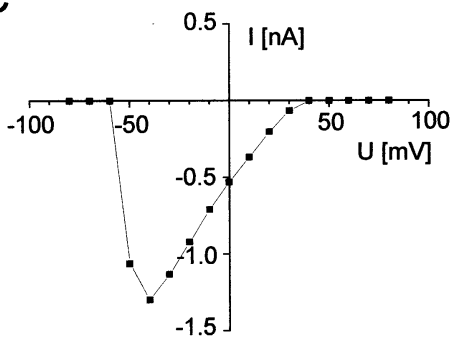

Fig. 6. Odorant responses in whole-cell current-clamp mode. (A) Response of an ORN (stage 52) to the mixture of all AA. (B) Response of an ORN (stage 51) to algae extract. (C) Activation curve of the voltage dependent sodium current in ORNs.

old for action potentials was approximately $-55 \mathrm{mV}$, which corresponded to the threshold of the voltagedependent sodium current determined in voltage-clamp experiments (Fig. 6C, observed in 15 ORNs).

Odorant responses of ORNs recorded in the wholecell configuration in the slice showed a pronounced rundown (Fig. 7, seen in 9 other ORNs).

None of the cells tested for volatile odorants responded to the mixtures $\mathrm{V}_{1}$ or $\mathrm{V}_{2}$.

\section{Discussion}

\subsection{Recording duration}

Recording times were markedly longer in the mucosa slice than in isolated ORNs. The average recording time of the successful recordings in the slice (Fig. 2D) was $44.5 \mathrm{~min}$, whereas the average recording time for isolated ORNs was just $6.4 \mathrm{~min}$ and the maximum rarely exceeded $15 \mathrm{~min}$. In the previous perforated-patch recordings of isolated ORNs (Vogler and Schild, 1999) the average recording duration was equal to or shorter than the average recording duration in the on-cell configuration in the slice. Isolated ORN recordings are
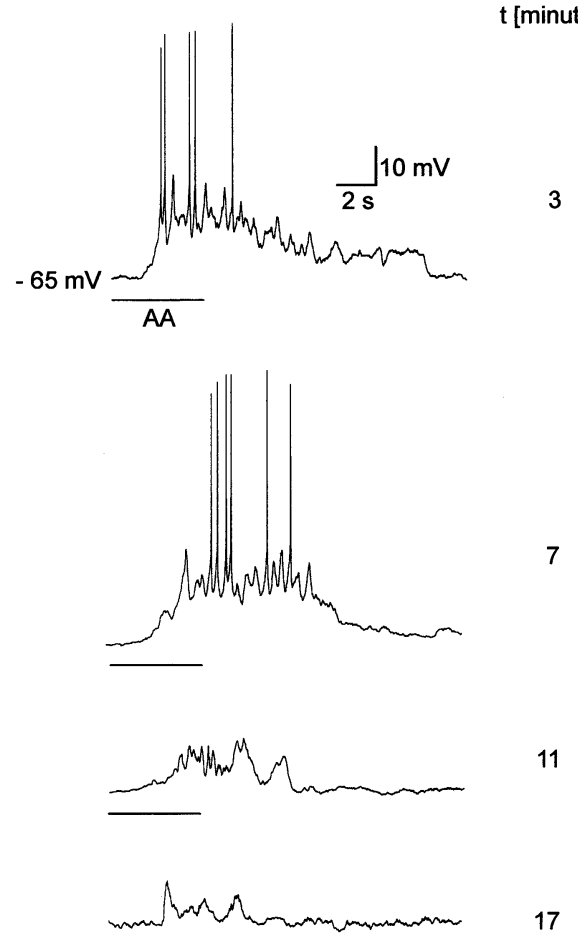

Fig. 7. Run-down of odorant responses over the time of an experiment. Run-down of the responses to the mixture of all AA of the ORN shown in Fig. 6A. Responses were taken at the times indicated at the right of the respective trace.

thus not the method of choice for testing many odorants on an individual cell.

\subsection{Odorant responses}

In the slice preparation $15.3 \%$ of the ORNs recorded in the on-cell configuration and $8.8 \%$ of the ORNs recorded in the whole-cell configuration showed responses to AA, i.e., on the average approximately $12 \%$, which is less than the previously reported $28 \%$ (Vogler and Schild, 1999). The lower frequency of odorant responses in the present slice preparation compared to that previously obtained (Vogler and Schild, 1999) is possibly due to the present ORNs being obtained from earlier stages of tadpole development. We presently recorded ORNs at stages $49-54$, while in the preceding study ORNs at stages $52-55$ were recorded.

All of the AA responses recorded in the whole-cell configuration in the slice showed a run-down (Fig. 7), similar to that reported for different species including Xenopus laevis (Lucero et al., 1992; Dionne, 1992; Lischka and Schild, 1993) recorded in isolated ORNs in the whole-cell configuration. On the other hand, responses recorded in the on-cell configuration and in the slice stayed stable until the end of the stimulus protocol (Fig. 5). 
These findings, together with the fact that the recording times of ORNs in the slice are longer than in isolated ORNs clearly indicate that the slice preparation combined with the on-cell configuration of the patch-clamp technique is the method of choice for testing many odorants on an individual ORN. Furthermore, on-cell recording of ORNs in the slice have the advantage, that their spontaneous activity is always sufficiently high (Fig. 4) to allow the detection of both excitatory and inhibitory responses.

The finding that 26 out of 35 ORNs recorded in the slice preparation responded to $\mathrm{SP}$, but not to AA suggests that AA are not the only stimuli of ORNs in the tadpole mucosa and indicates little overlap between the receptors for AA and those of at least some of the active components in the SP.

Disturbingly, 102 isolated ORNs tested in the on-cell recording mode did not respond to odorants at all. However, isolated ORNs recorded in the perforatedpatch mode responded to AA (Vogler and Schild, 1999). The lack of responsivity of isolated ORNs in the on-cell mode might have been caused by depolarized membrane potentials and partly inactivated sodium channels. ORNs, in the perforated-patch or whole-cell configuration held at a more negative potential would presumably not show this lack of responsivity. This interpretation is further supported by the fact that isolated ORNs recorded in the on-cell mode rarely showed spontaneous activity, whereas ORNs recorded in the on-cell mode in the slice were always spontaneously active. Taken together, isolated ORNs might have a less negative membrane potential than ORNs in the mucosa slice, and this would explain the lacking responsivity in on-cell recordings from isolated ORNs.

Volatile odorants, such as those contained in the mixtures $\mathrm{V}_{1}$ or $\mathrm{V}_{2}$, are reported to induce odorant responses in a large number of species including amphibia (Schild and Restrepo, 1998), in particular adult Xenopus laevis (air mucosa: Lischka and Schild, 1993; Zhainazarov and Ache, 1995; water mucosa: Iida and Kashiwayanagi, 1999). In the tadpole mucosa slice preparation, however, we never observed any response to volatile stimuli ( 0 out of 58 ORNs, mixtures $V_{1}$ or $\mathrm{V}_{2}$ ). It is possible that a stage-dependent expression of the corresponding receptor proteins might be responsible for this discrepancy.

In the previous perforated-patch recordings (stages 52-55), approximately half of the responses to single AA were inhibitory (Vogler and Schild, 1999), while in the present slice preparation, inhibitory responses to AA were never observed. This does not necessarily imply that ORNs of Xenopus laevis tadpoles do not exhibit inhibitory responses, because (1) an over-all excitation in response to a stimulus mixture does not exclude an inhibitory stimulus component and (2) only $12 \%$ of the ORNs tested responded to AA; the responses of these cells as well as the responses of the remaining $88 \%$ of ORNs to other stimuli are unknown. In comparison, inhibitory responses of single ORNs recorded in vivo are well established in catfish (Kang and Caprio, 1995), and the interaction of excitatory and inhibitory responses in the same receptor neuron has clearly been shown in lobster (Michel and Ache, 1994).

The possibility of inhibitory ORN responses in tadpole cannot be excluded; however, the occurrence of inhibitions in about $50 \%$ of the responsive isolated ORNs tested in the previous study deviates markedly from the present results of a complete absence of inhibitory responses. This striking difference could be the result of the presence of mucus on the mucosal surface of the slice and the intact compartmentalization of the ORNs. Immunocytochemistry experiments in this preparation revealed that there is a mucus layer of about $10 \mu \mathrm{m}$ (see also Hansen et al., 1998) into which cilia and microvilli protrude. The ionic composition of this mucus, in particular under our experimental conditions, is unknown. Furthermore, the exact localization of channel proteins and transporters in ORNs as well as the precise ion and protein composition of the mucus appear to be crucial in this context. If the $\mathrm{K}^{+}$ concentration in the mucus were markedly higher than in the extracellular space (reviewed in Schild and Restrepo, 1998), the activation of $\mathrm{K}^{+}$channels as described by Bacigalupo and collaborators (Morales et al., 1994, 1995) would lead to depolarizing receptor potentials in the slice, while in isolated ORNs the receptor potentials would be hyperpolarizing and thus inhibitory.

Finally, the isolated ORNs were dissociated by enzymatic treatment. If and how this affects the odorant responses has never been studied in detail. In the slice preparation this problem does not exist.

\section{Acknowledgements}

We thank Drs Caprio, Rössler and Zippel for critically reading the $\mathrm{ms}$ and providing a number of helpful comments. We also thank G. Federkeil and J. Kuduz for expert technical help.

\section{References}

Caprio J, Byrd RP. Electrophysiological evidence for acidic, basic, and neutral amino acid olfactory sites in the catfish. J Gen Physiol 1984;84:403-22.

Dionne VE. Chemosensory responses in isolated olfactory receptor neurons from Necturus maculosus. J Gen Physiol 1992;99:415-33.

Edwards FA, Konnerth A, Sakmann B, Takahashi T. A thin slice preparation for patch-clamp recordings from neurones of the mammalian central nervous system. Pflugers Arch 1989;414:60012. 
Frings S, Lindemann B. Current recording from sensory cilia of olfactory receptor cells in situ. I. The neuronal response to cyclic nucleotides. J Gen Physiol 1991;97:1-16.

Getchell TV. Functional properties of vertebrate olfactory receptor neurons. Physiol Rev 1986;66:772-807.

Hamill OP, Marty A, Neher E, Sakmann B, Sigworth FJ. Improved patch-clamp techniques for high-resolution current recording from cells and cell-free membrane patches. Pflugers Arch 1981;391:85100.

Hansen A, Reiss JO, Gentry CL, Burd GD. Ultrastructure of the olfactory organ in the clawed frog, Xenopus laevis, during larval development and metamorphosis. J Comp Neurol 1998;398:27388.

Iida A, Kashiwayanagi M. Responses of Xenopus laevis water nose to water-soluble and volatile odorants. J Gen Physiol 1999;114:8592.

Imanaka Y, Takeuchi H. Spiking properties of olfactory receptor cells in the slice preparation. Chem Senses 2001;26:1023-7.

Kang J, Caprio J. In vivo responses of single olfactory receptor neurons in the channel catfish, Ictalurus punctatus. J Neurophysiol 1995;73:172-7.

Lischka FW, Schild D. Effects of nitric oxide upon olfactory receptor neurones in Xenopus laevis. Neuroreport 1993;4:582-4.

Lucero MT, Horrigan FT, Gilly WF. Electrical responses to chemical stimulation of squid olfactory receptor cells. J Exp Biol 1992;162:231-49.

Lynch JW, Barry PH. Action potential initiated by single channels opening in a small neuron (rat olfactory receptor). Biophys $\mathrm{J}$ 1989;55:755-68.

Michel WC, Ache BW. Odor-evoked inhibition in primary olfactory receptor neurons. Chem Senses 1994;19:11-24.
Morales B, Ugarte G, Labarca P, Bacigalupo J. Inhibitory $\mathrm{K}^{+}$current activated by odorant in toad olfactory neurons. Proc R Soc Lond B 1994;257:235-42.

Morales B, Labarca P, Bacigalupo J. A ciliary $\mathrm{K}^{+}$conductance sensitive to charibdotoxin underlies inhibitory responses in toad olfactory receptor neurons. FEBS Lett 1995;359:41-4.

Nezlin LP, Schild D. Structure of the olfactory bulb in tadpoles of Xenopus laevis. Cell Tissue Res 2000;302:21-9.

Nieuwkoop PD, Faber J. Normal table of Xenopus laevis (Daudin). Amsterdam: Anonymous North Holland Company, 1956.

Schild D. A computer-controlled device for the application of odours to aquatic animals. J Electrophysiol Technol 1985;12:71-9.

Schild D, Gennerich A, Schultens HA. Microcontrollers as inexpensive pulse generators and parallel processors in electrophysiological experiments. Med Biol Eng Comput 1996;34:305-7.

Schild D, Restrepo D. Transduction mechanisms in vertebrate olfactory receptor cells. Physiol Rev 1998;78:429-66.

Sklar PB, Anholt RR, Snyder SH. The odorant-sensitive adenylate cyclase of olfactory receptor cells. Differential stimulation by distinct classes of odorants. J Biol Chem 1986;261:15538-43.

Taniguchi M, Kashiwayanagi M, Kurihara K. Intracellular dialysis of cyclic nucleotides induces inward currents in turtle vomeronasal receptor neurons. J Neurosci 1996;16:1239-46.

Vogler C, Schild D. Inhibitory and excitatory responses of olfactory receptor neurons of Xenopus laevis tadpoles to stimulation with amino acids. J Exp Biol 1999;202:997-1003.

Zhainazarov AB, Ache BW. Odor-induced currents in Xenopus olfactory receptor cells measured with perforated-patch recording. J Neurophysiol 1995;74:479-83. 